\title{
Excitation Mechanism of Extended Emission-Line Regions in Active Galaxies
}

\section{Ian Evans}

Smithsonian Astrophysical Observatory, 60 Garden Street, MS-27, Cambridge, MA 02138, USA

Anuradha Koratkar

Space Telescope Science Institute, 3700 San Martin Drive, Baltimore, $M D 21218$, USA

\section{Mark Allen and Zlatan Tsvetanov}

The Johns Hopkins University, Department of Physics and Astronomy, 3200 Charles Street, Baltimore, MD 21218, USA

\section{Michael Dopita}

Mount Stromlo and Siding Spring Observatories, Weston PO, ACT 2611, Australia

\begin{abstract}
Photoionization and shock models of the extended emissionline regions (EELRs) in active galaxies demonstrate that the optical emission lines alone are a poor discriminant of the excitation mechanism. Combining optical and UV data provides a discriminant between nuclear photoionization and autoionizing shock models. Hubble Space Telescope UV spectrophotometry of two Seyferts suggests that the EELRs in these objects are probably photoionized by the nucleus.
\end{abstract}

\section{Introduction}

Extended emission-line regions (EELRs) are often found in active galaxies. These regions of emission-line gas are distinct from the nuclear emission-line regions, and may or may not be morphologically connected to them. EELRs are observed in active galaxies with both broad and narrow emission-line nuclei, and in objects with and without strong radio jets. Although EELRs in active galaxies have been well observed, several fundamental questions about the mechanisms that excite these regions remain unresolved. Three key questions are:

1. What is/are the dominant ionization mechanism(s) in the EELRs of active galaxies?

2. Is the nuclear radiation field dominant, or are local ionization processes significant?

3. Is the primary ionization mechanism dependent on the type of object, or are the EELRs in all objects similar? 
Some of the pioneering models (e,g., Tadhunter, Robinson, \& Morganti 1989, Morganti et al. 1991) of EELRs attempted to reproduce the optical emission-line spectra of the optical filaments observed associated with the powerful radio galaxy Cen $\mathrm{A}$. These models invoke beamed photoionization from the nucleus. Although the models reproduce well the relative intensities of the strong optical emission lines, they do not predict correctly the observed ratio of the $\left[\mathrm{O}_{\mathrm{III}}\right] \lambda 4363 / \lambda 5007$ electron-temperature sensitive lines. This discrepancy between photoionization-model predictions and the measured [O III] emission-line ratios is observed in many other EELRs also.

More recent models completely eliminate the need for beamed photoionization, instead exciting the EELRs through local high-velocity (several hundred $\mathrm{km} \mathrm{s}^{-1}$ ) 'autoionizing' shocks that heat the local medium sufficiently that it reradiates in the ionizing UV and soft X-ray (Sutherland, Bicknell, \& Dopita 1993). Such models reproduce the observed optical emission-line strengths, including the $[0 \mathrm{III}] \lambda 4363 / \lambda 5007$ line ratio. The required shock velocities are fully consistent with the observed optical emission-line widths. The latter are sufficiently high that shocks arising from cloud-cloud collisions are inevitable.

\section{Model Predictions}

Shock models and beamed photoionization models are distinguished by the effects of the ionizing photon field. Beaming modifies the nuclear (non-thermal) photon field by geometrical dilution and absorption of soft photons by intervening material, producing a weak, hard photoionizing continuum that results in a high ionization state in the EELR gas. Low-velocity (non-autoionizing) shocks produce a 'classic' shock emission-line spectrum. High-velocity radiative (autoionizing) shocks produce UV and soft X-ray photons in the hot, shocked material; these photons then photoionize the surrounding medium. Because autoionizing-shock photoionization is local, it does not suffer from geometrical dilution or absorption in the same way as nuclear photoionization. The resulting ionization state in the EELR gas is therefore lower than would be expected from beamed nuclear photoionization.

All the excitation mechanisms predict very similar intensity ratios for the strong optical emission lines. However, they predict substantially different values of the $[\mathrm{O} \mathrm{III}] \lambda 4363 / \lambda 5007$ emission-line ratios. Nevertheless, although the $[\mathrm{O}$ III] ratio can be used to differentiate pure photoionization models, the ratio is not a good diagnostic for distinguishing between low-velocity and autoionizing shockheated gas. This is because the electron temperature in autoionizing shockexcited regions is lower than would be predicted from the shock velocity alone, due to emissivity averaging over the region photoionized by the shock.

By using the optical emission-line ratios to constrain the models, comparison of the observed and predicted intensity ratios of the strong UV emission lines is a very powerful discriminant amongst the models. Beamed photoionization models that reproduce the strong optical emission lines predict weak emission from the CII, CIII], and CIV UV lines. Most of the UV emission occurs in higher ionization-state lines, such as $\mathrm{NV}$ and $\mathrm{O}$ VI. Autoionizing shock models predict UV carbon line strengths that are 10-100 times stronger than the beamed photoionization models. 


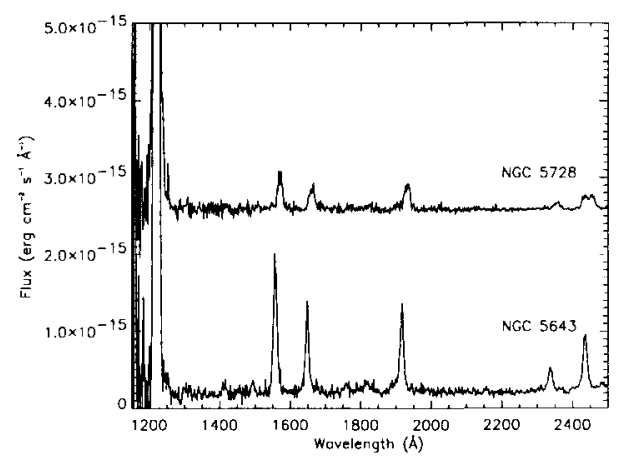

Figure 1. HST G160L spectra of the EELRs in NGC 5643 (bottom) and NGC 5728 (top). The NGC 5728 spectrum is shifted upward by $2.5 \times 10^{-15} \mathrm{ergs} \mathrm{s}^{-1} \mathrm{~cm}^{-2} \AA^{-1}$.

An excellent example of the differences between the beamed photoionization and autoionizing-shock model predictions is seen in M 87. Although the optical lines ratios are ambiguous, Hubble Space Telescope (HST) observations of the UV spectra reveal line intensity ratios that are readily explained by the autoionizing-shock model, but would be very difficult to reproduce using nuclear photoionization alone (Dopita et al. 1996).

\section{HST Spectrophotometry of EELRs in Seyferts}

Autoionizing-shock models apparently reproduce well the observed emission-line intensities in the EELRs in Cen A and M 87. However, these objects have powerful radio jets that are a source of mechanical energy input to the interstellar medium, and therefore high-velocity shocks may be expected in cloud-cloud collisions. Outflows in Seyfert galaxies may not be collimated to the same degree as radio jets, and the mechanical energy input to the EELRs may thus be much less than in the powerful radio-loud objects. Consequently, observations of EELRs in radio-loud objects alone cannot answer the three key questions posed above.

There are several Seyferts that have distinct EELRs visible from the ground for which we have obtained spatially resolved optical spectrophotometry. We have in addition with $H S T$ obtained UV spectrophotometry of the EELRs in two objects, NGC 5643 and NGC 5728.

For each galaxy, the Faint Object Spectrograph was used to obtain highresolution spectrophotometry of the EELR covering the spectral region $\sim 1150$ $2200 \AA$, with prism observations covering $\sim 1150-5400 \AA$ to tie the flux scale to ground-based optical data. A $1^{\prime \prime}$ square aperture was used to match the seeing for the ground-based optical data.

Comparison of ratios determined using accurate line-intensity measurements with a grid of models computed by Allen et al. (these proceedings) suggest that the EELRs observed are most compatible with photoionization-model predictions. The relative intensity of the $\left[\mathrm{O}_{\mathrm{III}}\right] \lambda 4363 / \lambda 5007$ line ratio indicates that the gas comprising both EELRs has electron temperatures of order 


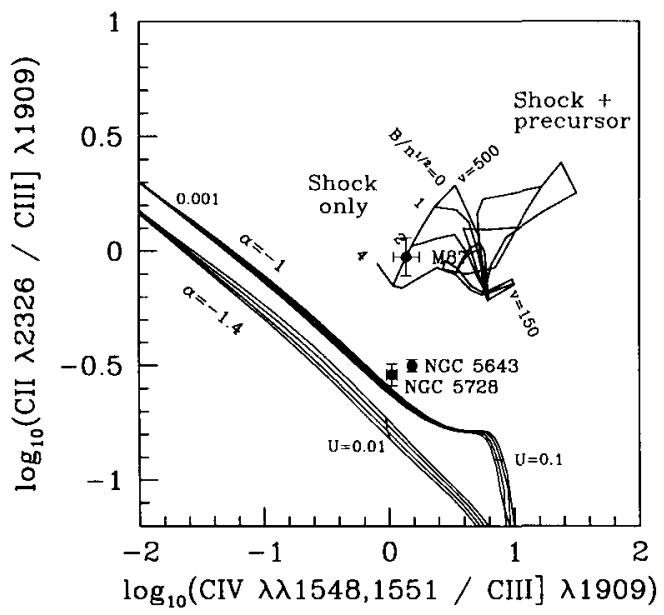

Figure 2. Plot of theoretical power-law photoionization and autoionizing-shock model line-ratio predictions, with the observed M 87 and Seyfert EELR line ratios plotted (courtesy Allen et al. 1996).

$1.5 \times 10^{4} \mathrm{~K}$, suggesting the possible presence of a low-velocity shock component. Shock velocities of order $100-200 \mathrm{~km} \mathrm{~s}^{-1}$ are consistent with the observed optical emission-line widths; such shocks would not be autoionizing.

\section{Conclusions}

The main conclusions of this work are: (1) The observations of the EELRs in the two Seyferts studied are not consistent with shock autoionization. (2) The sample is too small to determine whether shock autoionization is important in Seyfert EELRs in general; HST data for additional objects are required to resolve this question. (3) The UV data appear to be most consistent with photoionization. However, the combined UV and optical data indicate that neither pure photoionization nor pure shocks can explain all of the observed line ratios. A combination of photoionization and local low-velocity shocks appears to be needed to explain the observations. (4) Optical observations alone are not adequate to discriminate between ionization mechanisms; combined high-quality UV and optical data are essential.

\section{References}

Dopita, M.A., et al. 1996, in preparation.

Morganti, R., et al. 1991, MNRAS, 249, 91.

Sutherland, R. S., Bicknell, G. V., \& Dopita, M. A. 1993, ApJ, 414, 510.

Tadhunter, C. N., Robinson, A., \& Morganti, R. 1989, in Extranuclear Activity in Galaxies, ed. J. Meurs \& R. Fosbury, (Garching: ESO), 293. 